\title{
A note on "A multi-objective genetic algorithm for solving assembly line balancing problem"
}

\begin{abstract}
Assembly line balancing has a considerable place in industrial importance. Hence, a lot of researchers are interested in this subject and several papers have been published so far. Many exact, heuristic, metaheuristic, and hybrid approaches have been used to solve this type of problems. Recently Ponnanbalam et al. (Int J Adv Manuf Technol 16:341-352, 2000) have considered a multi-objective genetic algorithm utilizing several simple heuristic rules for solving the simple assembly line balancing problems, one of these rules was "rank positional weight (RPW)" originally published in Helgeson and Birnie (J Ind Eng 12(6):394-398, 1961). Through providing two possible justifications, this note suggests that the mentioned rule can be mistakenly utilized and some revisions in Ponnanbalam et al. (Int J Adv Manuf Technol 16:341-352, 2000) seem to be necessary.
\end{abstract}

Keyword: Assembly line balancing; Heuristic rules; Rank positional weight; Genetic algorithm 